\title{
Oncogenes, Tyrosine-Kinase
}

National Cancer Institute

\section{Source}

National Cancer Institute. Oncogenes, Tyrosine-Kinase. NCI Thesaurus. Code C18344.

Activated forms of tyrosine kinase family proto-oncogenes that contribute to the development of cancer. These proto-oncogenes encode tyrosine kinase proteins, which are enzymes that play a major role in mitogenic signaling by catalyzing the phosphorylation of tyrosine residues in proteins. Tyrosine kinase oncogenes exhibit aberrant gene expression, resulting in elevated levels of their protein products which disrupts mitotic checkpoints. 